\title{
Oligoadenylate synthetases-like is a prognostic biomarker and therapeutic target in pancreatic ductal adenocarcinoma
}

\author{
Shihong Chen ${ }^{1 \#}$, Zhijian Sun ${ }^{2,3 \#}$, Weizhu Zhao ${ }^{4}$, Mingyang Meng ${ }^{5}$, Wenyi Guo ${ }^{1}$, Dong Wu ${ }^{1}$, Qiang Shu ${ }^{2,3}$, \\ Jie Chai ${ }^{6}$, Lei Wang ${ }^{1}$
}

${ }^{1}$ Department of Pancreatic Surgery, General Surgery, Qilu Hospital, Cheeloo College of Medicine, Shandong University, Jinan, China; ${ }^{2}$ Department of Rheumatology, Qilu Hospital, Cheeloo College of Medicine, Shandong University, Jinan, China; ${ }^{3}$ Shandong Provincial Clinical Research Center for Immune Diseases and Gout, Qilu Hosptial, Jinan, China; ${ }^{4}$ Department of Radiology, Cancer Hospital Affiliated to Shandong First Medical University, Shandong Cancer Hospital and Institute, Jinan, China; ${ }^{5}$ Department of General Medicine, Xiangyang No. 1 People's Hospital, Hubei University of Medicine, Xiangyang, China; ${ }^{6}$ Department of Gastrointestinal Surgery, Cancer Hospital Affiliated to Shandong First Medical University, Shandong Cancer Hospital and Institute, Jinan, China

Contributions: (I) Conception and design: S Chen, W Zhao, J Chai; (II) Administrative support: L Wang, Q Shu; (III) Provision of study materials or patients: Z Sun, W Guo; (IV) Collection and assembly of data: D Wu; (V) Data analysis and interpretation: M Meng; (VI) Manuscript writing: All authors; (VII) Final approval of manuscript: All authors.

\#These authors contributed equally to this work.

Correspondence to: Jie Chai. Department of Gastrointestinal Surgery, Cancer Hospital Affiliated to Shandong First Medical University, Shandong Cancer Hospital and Institute, Jinan, China. Email: jchai@sdfmu.edu.cn; Lei Wang. Department of Pancreatic Surgery, General Surgery, Qilu Hospital, Cheeloo College of Medicine, Shandong University, Jinan 250012, China. Email: qlwanglei1102@163.com.

Background: Pancreatic ductal adenocarcinoma (PDAC) is fatal cancer that causes death. Early metastasis, resistance to chemotherapy, and lack of treatment contribute to a poor prognosis. Therefore, finding new therapeutic targets and biomarkers is a particularly urgent need to improve the survival of PDAC patients. Oligoadenylate synthetases-like (OASL), an antiviral enzyme produced by interferon (IFN), has been found to be associated with the occurrence and development of multiple cancers. However, its role in PDAC has been less well-studied. The value of OASL in PDAC was evaluated by bioinformatics and in vitro experiments.

Methods: The expression of OASL was evaluated using the Oncomine and Gene Expression Profiling Interactive Analysis (GEPIA) online websites. The survival time was also calculated by GEPIA. The correlation between OASL messenger RNA (mRNA) expression and immune infiltration was analyzed by the Tumor Immune Estimation Resource (TIMER) database. Clinical characteristics were revealed by The Cancer Genome Atlas (TCGA) data. A nomogram and forest plot were constructed based on univariate and multivariate Cox regression. Cell experiments [western blot assays, 3-(4,5-dimethylathiazol-2-yl)-2,5diphenyltetrazolium bromide (MTT) assays, transwell assays, flow cytometry assays] were used to verify the value of OASL in PDAC cells (Panc-1, Mia paca-2, and Aspc-1).

Results: A higher expression of OASL was observed in PDAC $(\mathrm{P}<0.05)$. Patients with increased expression of OASL had worse overall survival $(\mathrm{OS} ; \mathrm{P}<0.05)$ and disease-specific survival (DSS; $\mathrm{P}<0.05)$. The expression of OASL was correlated with $\mathrm{T}$ stage $(\mathrm{P}=0.030)$ and $\mathrm{N}$ stage $(\mathrm{P}=0.004)$, radiation therapy $(\mathrm{P}=0.013)$, primary therapy outcome $(\mathrm{P}<0.001)$, residual tumor $(\mathrm{P}=0.028)$, and tumor location $(\mathrm{P}=0.004)$ by univariate analysis, which also confirmed that OASL was an independent prognostic factor. Moreover, OASL expression was positively associated with neutrophils. In vitro experiments indicated that knockdown of OASL inhibited cell viability and invasion while increasing apoptosis rate.

Conclusions: High expression of OASL is associated with poor prognosis. Targeting OASL delays PDAC tumor progression in vitro. We highlight that OASL is a novel prognostic biomarker and therapeutic target of PDAC. 
Keywords: Oligoadenylate synthetases-like (OASL); pancreatic ductal adenocarcinoma (PDAC); prognosis; immune infiltration; TCGA

Submitted Nov 18, 2021. Accepted for publication Jan 05, 2022.

doi: $10.21037 / \mathrm{atm}-21-6618$

View this article at: https://dx.doi.org/10.21037/atm-21-6618

\section{Introduction}

Pancreatic cancer (PC) is a highly malignant digestive tract tumor characterized by strong invasiveness, a high recurrence rate, and poor prognosis (1). According to statistics, more than 500,000 people are diagnosed with PC annually (2). Pancreatic ductal adenocarcinoma (PDAC) is the most common pathological type of $\mathrm{PC}$ and causes more than $95 \%$ of PC patients' death (3). The prognosis of PDAC patients is unfavorable. The median survival time is only 6 months, whereas the 5 -year survival rate is below $9 \%$ (4). Surgery represents the only chance of cure for PDAC patients in the early stage; however, only about $20 \%$ have the opportunity to undergo an operation owing to the presence of metastasis at the time of diagnosis (5).

In recent years, targeted therapy has made some progress in PDAC, bringing new opportunities for treating PC. It has been found that the occurrence of PDAC is chiefly related to uncontrolled tumor suppressor genes and oncogenes, such as mutations in KRAS, TPP53, CDLM2A, SMAD4, EGFR, and $B R C A$. Several drugs have been developed for these therapeutic targets (6). However, due to chemotherapeutic resistance, many PDAC patients cannot benefit from the current targeted therapy (7). Therefore, it is vital to find new therapeutic targets and biomarkers to predict outcomes.

The oligoadenylate synthase (OAS) proteins, identified as enzymes sensing exogenous nucleic acid and initiating antiviral pathways, are induced by interferon (IFN). The 2'5 ' oligoadenylate synthetase-like (OASL) is a member of the OAS family. Unlike other members such as OAS1, OAS2, and OAS3, OASL lacks the activity of 2'-5' oligoadenylate synthetase activity. The function of OASL has been well characterized in different viral infection stages. In terms of tumor metastasis and growth, compared with wild-type mice, the OASL ${ }^{-1}$ mice showed more produced more type I interferon-regulating transcription factor (IRF7), which inhibited tumor progression by enhancing the effector function of CD8 T + cells and NK cells. It is suggested that OASL can play an immune-related role in cancers $(8,9)$. However, these studies are only limited to how OASL regulates the progression of tumors through the immune system. The role of OASL in tumor itself is rarely reported. Studies have shown that OASL is closely related to the proliferation of various tumors (10). In addition, OASL has been identified as a pivotal gene of trastuzumab-resistant gastric cancer (11). It has a good prognostic value in breast cancer (12) and enhances the efficacy of drug therapy in lung cancer (13). In conclusion, OASL may play a role as a proto-oncogene in tumor cells and promote the occurrence and development of cancer. However, the function of OASL in PDAC is rarely concerned.

Our research not only confirmed that it could be used as a prognostic biomarker for PDAC, but also explored its role in the occurrence and development of PDAC and further verified that OASL might be a potential therapeutic target in the future.

We present the following article in accordance with the REMARK reporting checklist (available at https://atm. amegroups.com/article/view/10.21037/atm-21-6618/rc).

\section{Methods}

\section{Baseline information}

Data were extracted from The Cancer Genome Atlas (TCGA) database and retrospectively analyzed by $\mathrm{R}$ software (version 3.6.3; https://cran.r-project.org/bin/ windows/base/old/3.6.3/). The RNAseq data of fragments per kilobase per million (FPKM) format in PDAC were derived from TCGA, while $\log 2$ transformation was performed. The molecule was OASL (ENSG00000135114). The study was conducted in accordance with the Declaration of Helsinki (as revised in 2013).

\section{The expression of OASL and clinical characteristics in PDAC}

The expression of OASL in a variety of cancers was analyzed using the Oncomine database (https://www. oncomine.org/resource/login.html), and its expression in 
PDAC was analyzed using the Gene Expression Profiling Interactive Analysis (GEPIA) database (http://gepia.cancerpku.cn/index.html) $(14,15)$. The ggplot2 package and basic package were used to reveal the relationship between OASL and clinical features. High and low expression of OASL was used as independent variable types. The dependent variable was clinical characteristics.

\section{Receiver operating characteristic analysis}

The pROC and ggplot2 package was used for visualization. The UCSC XENA (https://xenabrowser.net/datapages/) RNA seq data in TPM format of TCGA and GTEX uniformly were processed by the Toil process (16). Data were compared between samples after $\log 2$ conversion.

\section{Kaplan-Meier survival curve}

The survminer package and survival package were used for survival analysis visualization and analysis. We performed $\log 2$ conversion of RNAseq data in FKM format in TCGA. We then verified the results using the survival module in GEPIA. The parameter settings were as follows: gene was OASL, group cutoff was median, the primary endpoint including overall survival (OS), disease-specific survival (DSS), and disease-free survival (DFS), were used as indicators to evaluate the correlation between OASL expression and prognosis.

\section{Gene Set Enrichment Analysis (GSEA)}

GSEA was used to associate genes with possible pathways. A false discovery rate (FDR) of $<0.25$ and $\mathrm{P}$ adjust $<0.05$ were used as the criteria for judging statistically significant. We chose the enrichment pathways with obvious statistical significance.

\section{TIMER database analysis}

Tumor Immune Estimation Resource (TIMER; https:// cistrome.shinyapps.io/timer/) is a public resource widely utilized to assess immune infiltration in multiple cancers from the TCGA database (17). The immune correlation module was used for analysis.

\section{Cell culture and transfection}

We used 3 human PDAC cells for in vitro experiments.
All cell lines were purchased from Procell Life Science \& Technology (Hyderabad, India) and authenticated by short tandem repeat (STR) profiling. All cell lines were cultured with Dulbecco's modified Eagle medium (DMEM; Thermo Fisher Scientific, Shanghai, China) and incubated at $37{ }^{\circ} \mathrm{C}$ with $5 \% \mathrm{CO}_{2}$. Interference of OASL was performed using small interfering RNA (si-OASL-NC; si-OASL-1, siOASL-2, and si-OASL-3) sequences synthesized by Tsingke Biotechnology (Beijing, China), the sequences are as follows: si-OASL-1 (5'-GTGAAACATCGGCCAACTA-3'), siOASL-2 (5'-CATCACGGTCACCATTGT-3'), si-OASL-3 (5'-GGTGGTCCTGGAAATTTCT-3'). Lipofectamine 3000 (Invitrogen, Shanghai, China) was used for transfection. Relevant experiments were carried out $48 \mathrm{~h}$ after transfection.

\section{MTT assay}

Cell viability was detected by 3-(4,5-dimethylathiazol-2yl)-2,5-diphenyltetrazolium bromide (MTT) assay. About $5 \times 10^{3}$ cells were seeded on 96-well plates. We added $20 \mu \mathrm{L}$ MTT solution followed by incubation for $4 \mathrm{~h}$ at each point $(24,48,72$, and $96 \mathrm{~h})$. The medium was aspirated, and $150 \mu \mathrm{L}$ of dimethyl sulfoxide (DMSO) was added to each well to disrupt the cells and dissolve the intracellular MTT dyes. Last, the absorbance was measured at $490 \mathrm{~nm}$ on a microplate reader. The MTT assays were performed in triplicate (for each experiment) and 3 independent biological repeats.

\section{Transwell assay}

Cell migration was detected by transwell assay. We seeded $1.5 \times 10^{4}$ cells into the upper chamber followed by culture with a serum-free medium. A total of $0.75 \mathrm{~mL}$ serumcontaining medium was added to the lower chambers. After incubation for $24 \mathrm{~h}$, the invaded cells were fixed with paraformaldehyde and stained with crystal violet and photographed in 3 random fields under a microscope $(10 \times 10)$. Each experiment was repeated 3 times.

\section{Flow cytometric analysis}

Apoptosis was detected by annexin $\mathrm{V}$ apoptosis kit (Vazyme, Jiangsu, China). Fluorescence-activated cell sorting (FACS) was performed on a BD Accuri ${ }^{\circledR}$ C6 Plus [Becton, Dickinson, and Co. (BD) Biosciences, Franklin Lakes, NJ, USA] and analyzed by FlowJo software (https://www. flowjo.com/). In a nutshell, $1 \times 10^{6}$ cells were collected and 
resuspended after adding $100 \mu \mathrm{L}$ binding buffer. Next, $100 \mu \mathrm{L}$ binding buffer containing $5 \mu \mathrm{L}$ annexin V-FIFC and $5 \mu \mathrm{L}$ propidium iodide (PI) staining solution was added. Last, $400 \mu \mathrm{L}$ binding buffer was added to the culture tube and analyzed within $1 \mathrm{~h}$ using flow cytometry.

\section{Western blot}

Total protein was obtained utilizing radioimmunoprecipitation assay (RIPA) lysis buffer (Servicebio, Wuhan, China). Protein concentration was quantified using the bicinchoninic (BCA) assay (Solarbio, Beijing, China). After adding loading buffer, samples were boiled for $5 \mathrm{~min}$. Then, $20 \mu \mathrm{g}$ protein was added to each lane, divided by $8-15 \%$ sodium dodecyl sulfatepolyacrylamide gel electrophoresis (SDS-PAGE), and then transferred to polyvinylidene fluoride (PVDF) membranes, which were blocked with 5\% no-fat dry milk in Tris-buffered saline with $0.1 \%$ Tween 20 (TBST) for $2 \mathrm{~h}$. The diluted primary antibodies against OASL $(1: 1,000)$ and glyceraldehyde 3-phosphate dehydrogenase (GADPH; 1:10,000) were incubated with the membrane overnight at $4{ }^{\circ} \mathrm{C}$. After cleaning with TBST for $10 \mathrm{~min}$, it was incubated with corresponding antibodies for $2 \mathrm{~h}$ and the membrane was cleaned 3 times with TBST. Last, electrochemiluminescence (ECL, Thermo, China) was applied for visualizing the results.

\section{Statistical analysis}

Part of the statistical analysis was carried out by default by network resources, and the others were carried out by $\mathrm{R}$ software and basic package (ggplot2, pROC, survminer, survival, rms). The Student's $t$-test was used for comparison between the 2 groups (si-OASL-NC $v s$. si-OASL-1, siOASL-NC $v s$. si-OASL-2, si-OASL-NC $v s$. si-OASL-3). Only $\mathrm{P}<0.05$ was considered statistically significant.

\section{Results}

\section{Clinical characteristics}

Participants' demographics and clinical variables related to PDAC are shown in Table 1. A total of 178 patients were involved in this study. The T stage included $7 \mathrm{~T} 1$ stage (4\%), $24 \mathrm{~T} 2$ stage (13.6\%), $142 \mathrm{~T} 3$ stage (13.6\%), and $3 \mathrm{~T} 4$ stage $(1.7 \%)$. The $\mathrm{N}$ stage included $50 \mathrm{~N} 0$ stage $(28.9 \%)$, and $23 \mathrm{~N} 1$ stage $(71.1 \%)$. The $\mathrm{M}$ stage included $79 \mathrm{M} 0$ stage (94\%) and $5 \mathrm{M} 1$ stage (6\%). In the pathologic stage, stage I, stage II, stage III, stage IV were 21 cases (12\%), 146 cases (83.4\%), 3 cases $(1.7 \%)$, and 5 cases (2.9\%), respectively.
The median age was 65 years, ranging from 57 to 73 years. Other clinical information is displayed in Table 1.

\section{OASL was bighly expressed in PDAC}

The Oncomine database was searched to obtain OASL expression levels in order to generally indicate the OASL in normal tissues and cancer. The expression of OASL was increased in PDAC and other cancers (Figure 1A). The GEPIA database was used for further verification $(\mathrm{P}<0.05$, Figure $1 B$ ). The area under the curve (AUC) of OASL was 0.984 (Figure 1C).

\section{Correlation between OASL expression and clinical characteristics}

The 178 PDAC patients were divided into 2 groups according to the median expression of OASL. The P value of $\mathrm{T}$ stage, pathologic stage, and histologic grade was 0.010 , 0.007 , and $<0.001$, respectively (Table 2). Logistics regression analysis of PDAC showed that the $\mathrm{P}$ value of T stage (T1\&T2 vs. T3\&T4) was 0.004 , the $\mathrm{P}$ value of pathologic stage (stage I vs. stage II) was 0.005 (Figure 2, Table 3).

\section{High expression of OASL associated with poor prognosis}

To further confirm the prognostic value of OASL, participants were divided into high and low groups according to the median expression of OASL. The results showed that high expression of OASL was significantly associated with poor OS $(\mathrm{P}<0.01)$ and DSS $(\mathrm{P}<0.01)$ in PDAC (Figure $3 A, 3 B$ ). We used the survival analysis module of GEPIA to verify the prognostic information of OASL in TCGA. The results of OS were consistent $(\mathrm{P}<0.01)$ (Figure 3C), while there was no significant difference in DFS (Figure 3D). Next, univariate and multivariate Cox regression analysis were used to explore the prognostic factors of PDAC. Besides, radiation therapy, primary therapy outcome and histologic grade also influenced tumor progression $(\mathrm{P}<0.05)($ Table 4$)$. The prognostic nomogram (Figure 4) and forest plot (Figure 5) were constructed based on Cox regression.

\section{OASL-related patbways based on GSEA and correlated with immune infiltration}

The OASL-related signaling pathways based on GSEA were enriched in OASL expression phenotypes. The top 
Table 1 Characteristics of patients with PDAC (TCGA)

\begin{tabular}{|c|c|c|}
\hline Characteristic & Levels & Overall $(n=178)$ \\
\hline \multirow[t]{4}{*}{ T stage, $\mathrm{n}(\%)$} & $\mathrm{T} 1$ & $7(4.0)$ \\
\hline & $\mathrm{T} 2$ & $24(13.6)$ \\
\hline & T3 & $142(80.7)$ \\
\hline & $\mathrm{T} 4$ & $3(1.7)$ \\
\hline \multirow[t]{2}{*}{ N stage, n (\%) } & NO & $50(28.9)$ \\
\hline & $\mathrm{N} 1$ & $123(71.1)$ \\
\hline \multirow[t]{2}{*}{ M stage, n (\%) } & MO & $79(94.0)$ \\
\hline & M1 & $5(6.0)$ \\
\hline \multirow[t]{4}{*}{ Pathologic stage, n (\%) } & Stage I & $21(12.0)$ \\
\hline & Stage II & $146(83.4)$ \\
\hline & Stage III & $3(1.7)$ \\
\hline & Stage IV & $5(2.9)$ \\
\hline \multirow[t]{2}{*}{ Radiation therapy, n (\%) } & No & $118(72.4)$ \\
\hline & Yes & $45(27.6)$ \\
\hline \multirow{4}{*}{$\begin{array}{l}\text { Primary therapy } \\
\text { outcome, n (\%) }\end{array}$} & PD & $49(35.3)$ \\
\hline & SD & $9(6.5)$ \\
\hline & PR & $10(7.2)$ \\
\hline & CR & $71(51.1)$ \\
\hline \multirow[t]{2}{*}{ Gender, n (\%) } & Female & $80(44.9)$ \\
\hline & Male & $98(55.1)$ \\
\hline \multirow[t]{2}{*}{ Age, n (\%) } & $\leq 65$ & 93 (52.2) \\
\hline & $>65$ & $85(47.8)$ \\
\hline \multirow[t]{3}{*}{ Residual tumor, n (\%) } & Ro & $107(65.2)$ \\
\hline & $\mathrm{R} 1$ & $52(31.7)$ \\
\hline & $\mathrm{R} 2$ & $5(3.0)$ \\
\hline \multirow[t]{4}{*}{ Histologic grade, n (\%) } & G1 & $31(17.6)$ \\
\hline & G2 & $95(54.0)$ \\
\hline & G3 & $48(27.3)$ \\
\hline & G4 & $2(1.1)$ \\
\hline \multirow{2}{*}{$\begin{array}{l}\text { Anatomic neoplasm } \\
\text { subdivision, } \mathrm{n}(\%)\end{array}$} & Head of pancreas & $138(77.5)$ \\
\hline & Other & $40(22.5)$ \\
\hline \multirow[t]{2}{*}{ Smoker, n (\%) } & No & $65(45.1)$ \\
\hline & Yes & $79(54.9)$ \\
\hline
\end{tabular}

Table 1 (continued)
Table 1 (continued)

\begin{tabular}{lcc}
\hline Characteristic & Levels & Overall $(\mathrm{n}=178)$ \\
\hline Alcohol history, n (\%) & No & $65(39.2)$ \\
& Yes & $101(60.8)$ \\
$\begin{array}{l}\text { History of diabetes, n } \\
\text { (\%) }\end{array}$ & No & $108(74.0)$ \\
& Yes & $38(26.0)$ \\
History of chronic & No & $128(90.8)$ \\
pancreatitis, n (\%) & Yes & $13(9.2)$ \\
& No & $47(42.7)$ \\
Family history of cancer, & & $63(57.3)$ \\
$\mathrm{n}(\%)$ & Yes & $86(48.3)$ \\
& Alive & $92(51.7)$ \\
OS event, n (\%) & Dead & $100(58.1)$ \\
Age, median [IQR] & Alive & $72(41.9)$ \\
PDAC, pancreatic ductal adenocarcinoma; TCGA, The Cancer \\
survival; PFI, progression-free interval; IQR, interquartile range.
\end{tabular}

5 pathways with notable statistical significance were GPCRligand binding, Neuronal system, Class A/1 (rhodopsinlike receptors), G-Alpha/1 signaling events, and Leishmania infection (Figure 6). Next, we found that OASL expression is significantly correlated with neutrophil in the TIMER database ( $\mathrm{r}=0.189, \mathrm{P}=1.32 \mathrm{e}-02$; Figure $7 A$ ). In addition, the scan module was used to test the effect of different copy states of OASL on immune infiltration (Figure 7B).

\section{Knockdown of OASL inbibits cells proliferation, invasion, and promotes apoptosis of $P D A C$}

Subsequently, to explore the effect of OASL interference, we performed at least 3 independent cellular experiments. We employed chemically synthesized siRNA to knock down the expression of OASL. The western blot assays showed siOASL-2 and si-OASL-3 have higher efficiency (Figure 8A). The MTT assays demonstrated that cell viability was sharply 

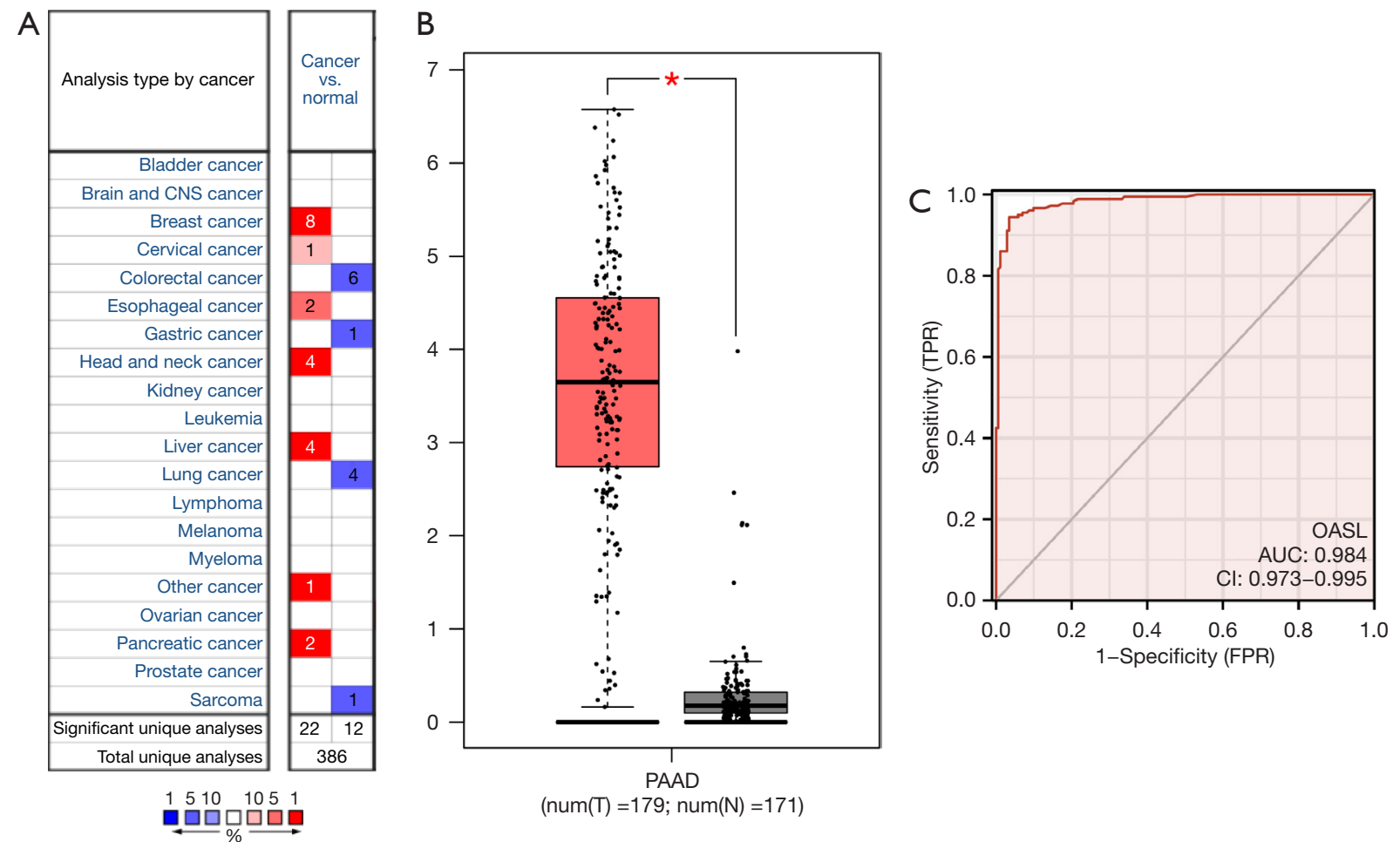

Figure 1 OASL expression is significantly higher in PDAC than in normal tissues. (A) OASL expression in different human cancer tissues compared with normal tissues by the Oncomine database. (B) OASL expression in PDAC is higher than adjacent normal tissues in GEPIA. (C) ROC curve. *, $\mathrm{P}<0.05$. CNS, central nervous system; PAAD, pancreatic adenocarcinoma; FPR, false positive rate; OASL, oligoadenylate synthetases-like; PDAC, pancreatic ductal adenocarcinoma; GEPIA, Gene Expression Profile Interactive Analysis; ROC, receiver operating characteristic.

reduced in si-OASL groups (Figure $8 B$ ). Similarly, the Transwell assay demonstrated that cells migration decreased significantly after OASL knockdown (Figure 8C). Flow cytometry showed that knockdown of OASKL promoted PDAC cells apoptosis (Figure 8D). These results confirm that OASL can be used as a therapeutic target to delay the malignant biological behavior of PDAC.

\section{Discussion}

The mortality of PC, in particular PDAC, is high (18). Due to the specific anatomical location of the pancreas, most patients are diagnosed at an advanced stage and have surpassed their only opportunity for a cure by surgical resection (19). Moreover, the aggressive local invasion and metastatic progression of PDAC are associated with a poor prognosis (20). Currently, TNM stage and hematologic markers (CA199, CEA, CA125) are used to evaluate the prognosis of patients. However, its effectiveness and accuracy are not high (21). Therefore, there is urgently needed for developing effective biological targets for the prediction and treatment of PDAC.

The OAS families, of which OASL is a member, have been found correlated with multiple diseases, including infections, autoimmune disorders, and cancers (22-24). In particular, it has been reported that OASL is significantly related to tumor proliferation (10). Our data highlighted the prognostic value and therapeutic target of OASL in PDCA. In this study, the expression of OASL and clinical characteristics were intimately connected. More importantly, patients with high expression of OASL had shorter OS and DSS. In addition, OASL expression was an independent prognostic factor of PADC patients' prognosis. Next, based on GSEA, OASL was related to pathways including GPCR-ligand binding, Neuronal system, Class A/1 (rhodopsin-like receptors), G-Alpha/1 signaling events, and Leishmania infection. In the accurate prognosis prediction of clear cell carcinoma, OASL was identified as a novel gene (25). In addition, OASL could be used as the key to evaluation of the sensitivity of cisplatin chemotherapy 
Table 2 Correlation between OASL expression and clinical characteristics of PDAC

\begin{tabular}{|c|c|c|c|}
\hline Characteristic & $\begin{array}{c}\text { Low expression } \\
\text { of OASL }\end{array}$ & $\begin{array}{l}\text { High expression } \\
\text { of OASL }\end{array}$ & $P$ value \\
\hline $\mathrm{n}$ & 89 & 89 & \\
\hline T stage, n (\%) & & & 0.010 \\
\hline $\mathrm{T} 1$ & $6(3.4)$ & $1(0.6)$ & \\
\hline $\mathrm{T} 2$ & $17(9.7)$ & $7(4.0)$ & \\
\hline T3 & $62(35.2)$ & $80(45.5)$ & \\
\hline $\mathrm{T} 4$ & $2(1.1)$ & $1(0.6)$ & \\
\hline $\mathrm{N}$ stage, n (\%) & & & 0.119 \\
\hline NO & $30(17.3)$ & $20(11.6)$ & \\
\hline $\mathrm{N} 1$ & $56(32.4)$ & $67(38.7)$ & \\
\hline M stage, n (\%) & & & 1.000 \\
\hline Mo & $39(46.4)$ & $40(47.6)$ & \\
\hline M1 & $2(2.4)$ & $3(3.6)$ & \\
\hline \multicolumn{2}{|c|}{ Pathologic stage, n (\%) } & & 0.007 \\
\hline Stage I & $17(9.7)$ & $4(2.3)$ & \\
\hline Stage II & $66(37.7)$ & $80(45.7)$ & \\
\hline Stage III & $2(1.1)$ & $1(0.6)$ & \\
\hline Stage IV & $2(1.1)$ & $3(1.7)$ & \\
\hline \multicolumn{2}{|c|}{ Radiation therapy, n (\%) } & & 0.961 \\
\hline No & $58(35.6)$ & $60(36.8)$ & \\
\hline Yes & $23(14.1)$ & $22(13.5)$ & \\
\hline Gender, n (\%) & & & 1.000 \\
\hline Female & $40(22.5)$ & $40(22.5)$ & \\
\hline Male & $49(27.5)$ & $49(27.5)$ & \\
\hline Race, n (\%) & & & 0.112 \\
\hline Asian & $3(1.7)$ & $8(4.6)$ & \\
\hline $\begin{array}{l}\text { Black or African } \\
\text { American }\end{array}$ & $5(2.9)$ & $1(0.6)$ & \\
\hline White & $79(45.4)$ & $78(44.8)$ & \\
\hline Age, n (\%) & & & 0.072 \\
\hline$\leq 65$ & $53(29.8)$ & $40(22.5)$ & \\
\hline$>65$ & $36(20.2)$ & $49(27.5)$ & \\
\hline
\end{tabular}

Table 2 (continued)
Table 2 (continued)

\begin{tabular}{|c|c|c|c|}
\hline Characteristic & $\begin{array}{c}\text { Low expression } \\
\text { of OASL }\end{array}$ & $\begin{array}{l}\text { High expression } \\
\text { of OASL }\end{array}$ & $P$ value \\
\hline \multicolumn{3}{|l|}{ Residual tumor, n (\%) } & 0.139 \\
\hline $\mathrm{RO}$ & $61(37.2)$ & $46(28.0)$ & \\
\hline $\mathrm{R} 1$ & $21(12.8)$ & $31(18.9)$ & \\
\hline $\mathrm{R} 2$ & $3(1.8)$ & $2(1.2)$ & \\
\hline \multicolumn{3}{|l|}{ Histologic grade, n (\%) } & $<0.001$ \\
\hline G1 & $25(14.2)$ & $6(3.4)$ & \\
\hline G2 & $39(22.2)$ & $56(31.8)$ & \\
\hline G3 & $21(11.9)$ & $27(15.3)$ & \\
\hline G4 & $2(1.1)$ & $0(0.0)$ & \\
\hline \multicolumn{3}{|l|}{ Smoker, n (\%) } & 0.248 \\
\hline No & $36(25.0)$ & $29(20.1)$ & \\
\hline Yes & $35(24.3)$ & $44(30.6)$ & \\
\hline \multicolumn{3}{|l|}{ Alcohol history, n (\%) } & 1.000 \\
\hline No & $32(19.3)$ & $33(19.9)$ & \\
\hline Yes & $51(30.7)$ & $50(30.1)$ & \\
\hline \multicolumn{3}{|l|}{$\begin{array}{l}\text { History of diabetes, } \\
\mathrm{n}(\%)\end{array}$} & 1.000 \\
\hline No & $53(36.3)$ & $55(37.7)$ & \\
\hline Yes & $18(12.3)$ & $20(13.7)$ & \\
\hline \multicolumn{3}{|c|}{ History of chronic pancreatitis, n (\%) } & 0.979 \\
\hline No & $65(46.1)$ & $63(44.7)$ & \\
\hline Yes & $6(4.3)$ & $7(5.0)$ & \\
\hline Age, mean \pm SD & $63.47 \pm 10.72$ & $66.02 \pm 10.79$ & 0.115 \\
\hline
\end{tabular}

in cervical cancer (26). Targeted OASL can improve drug efficacy in lung cancer (13). These studies indicate that OASL plays an essential role in the occurrence of cancers.

Previous studies have confirmed that the malignant progression of PDAC is highly associated with the tumor microenvironment $(27,28)$. We found that OASL was closely related to neutrophil infiltration, which was consistent with prior studies $(12,29)$. Neutrophils secrete a cocktail of 

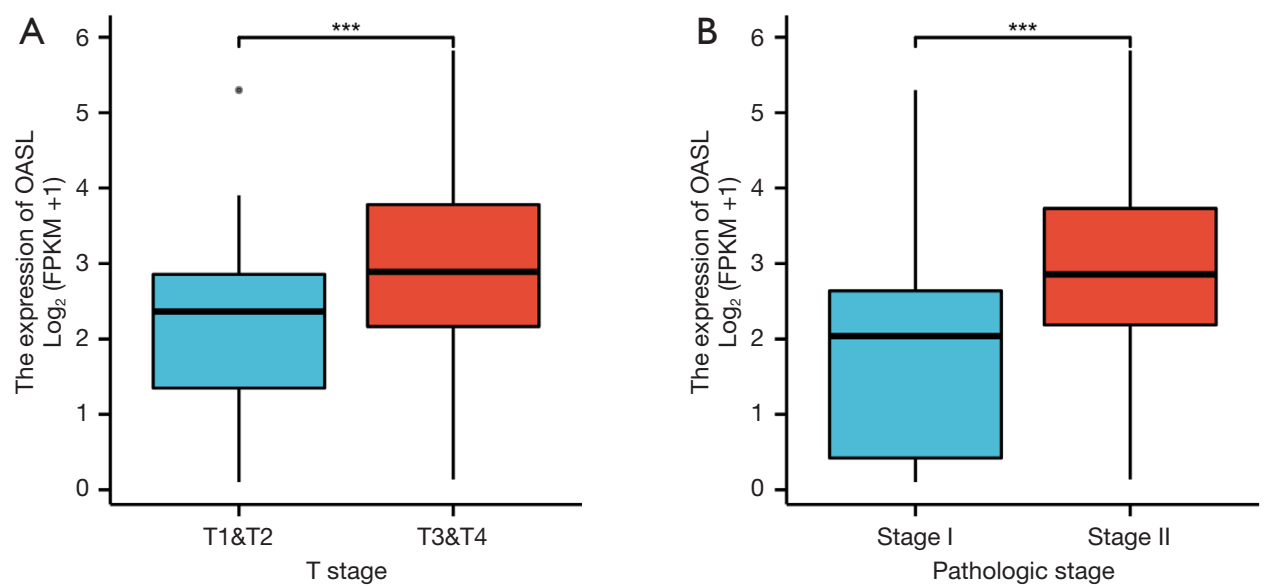

Figure 2 OASL expression in PDAC was associated with clinical characteristics. (A) T stage. (B) Pathologic stage. ${ }^{* * *}$, P<0.001. OASL, oligoadenylate synthetases-like; PDAC, pancreatic ductal adenocarcinoma.

Table 3 OASL expression was associated with clinical characteristics of PDAC (logistic regression)

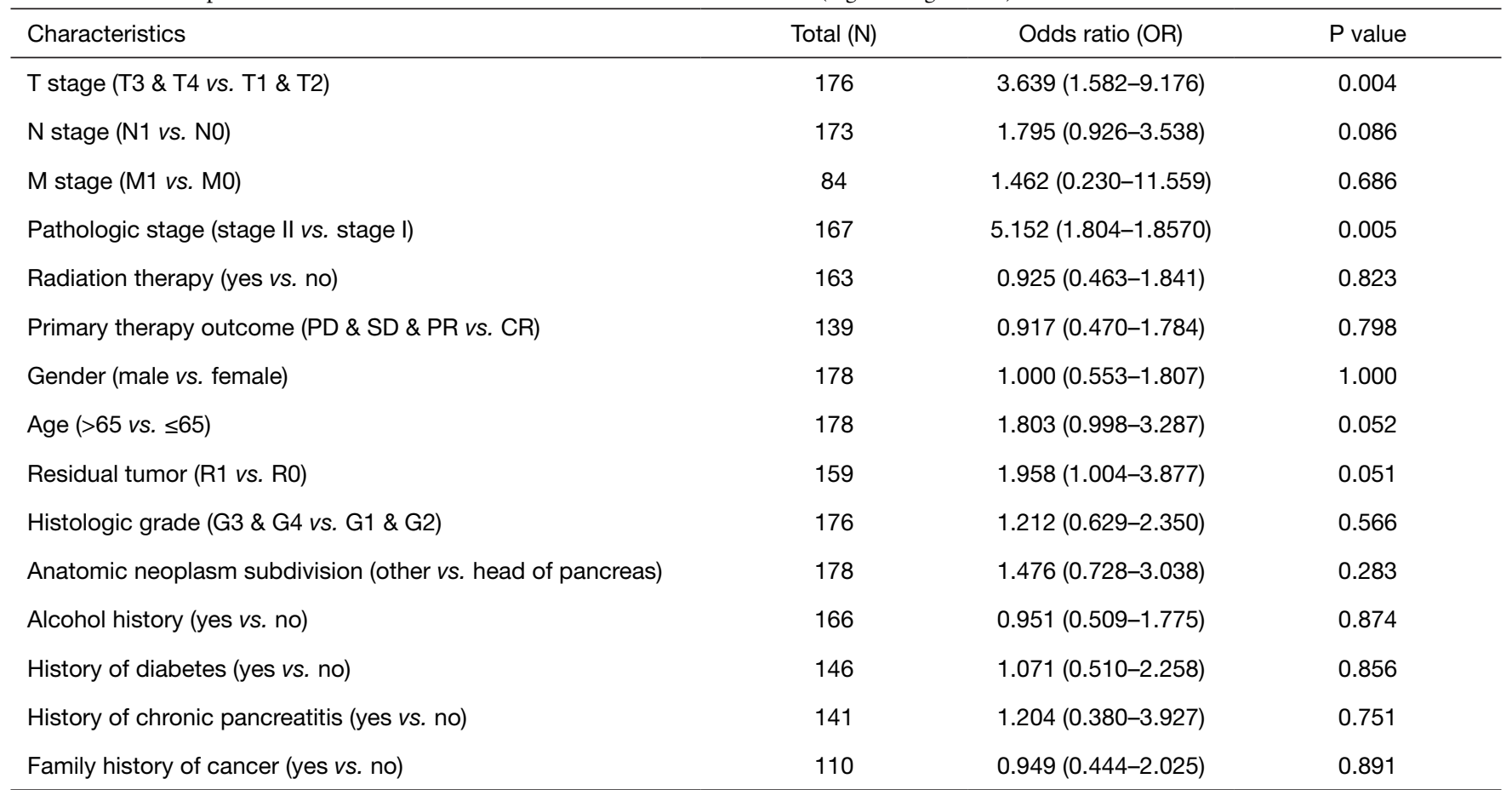

OASL, oligoadenylate synthetases-like; PDAC, pancreatic ductal adenocarcinoma; PD, progressive disease; SD, stable disease; PR, partial remission; CR, complete remission.

tumor-promoting factors, such as hepatocyte growth factor (HGF), matrix metalloproteinases (MMPs), reactive oxygen species (ROS), and promoted tumor angiogenesis, invasion, and metastasis (30). Moreover, some chemokines produced by neutrophils also lead to tumorigeneses, such as CCL2, CCL3, CCL19, and CCL 20 (31). We speculate that OASL may promote the function of neutrophils recruited to the tumor site, fail to mount an anti-tumor response, and thus lead to PDAC progression (32).

High invasiveness is the main cause of death in PDAC patients. The enhancement of cell viability, invasion, and migration are the main factors leading to tumor recurrence 

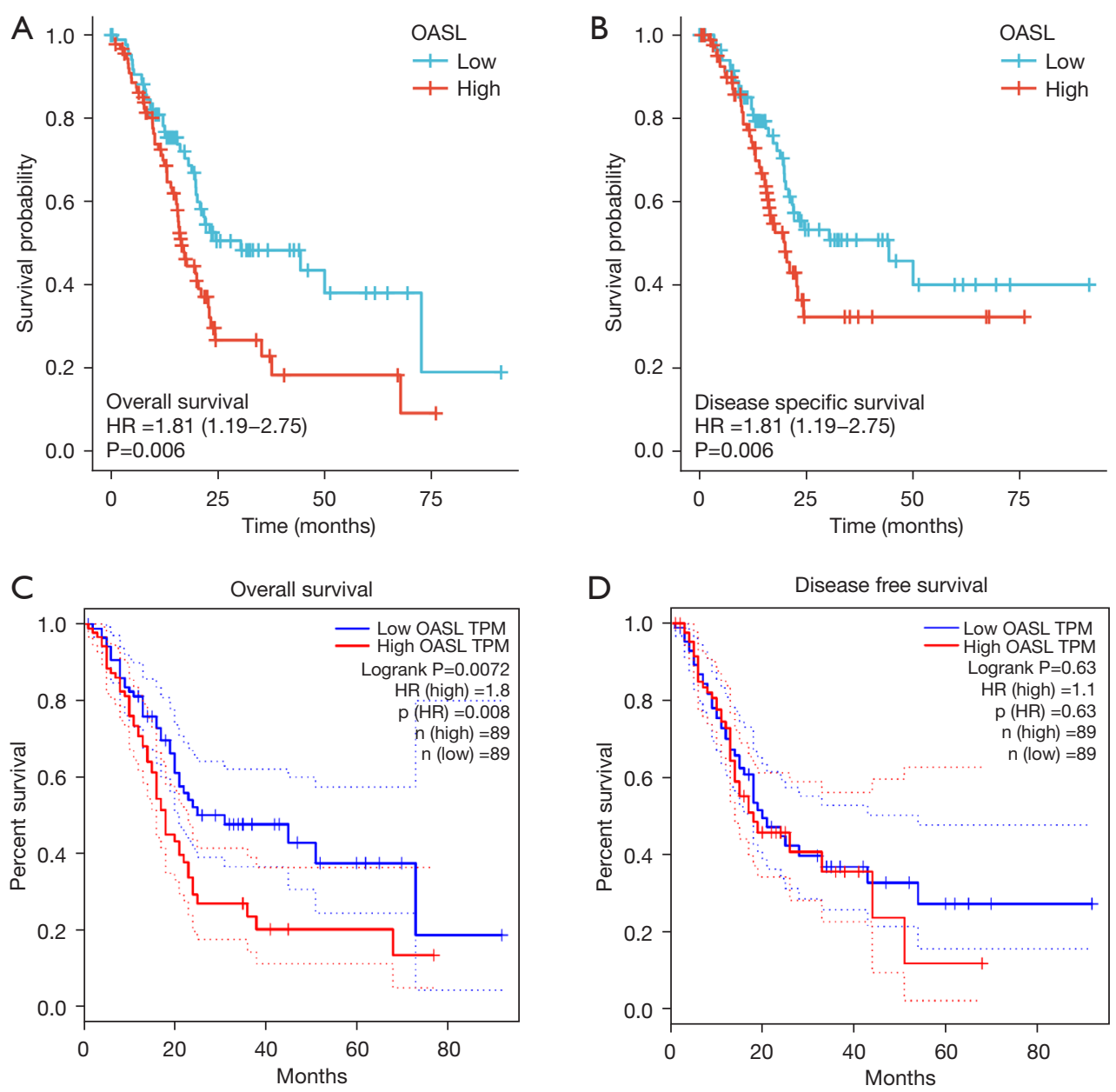

Figure 3 The Kaplan-Meier survival curves for different OASL levels. (A,B) The OS and DSS curves of PDAC patients with high vs. low OASL expression in TCGA database. (C,D) Survival curves of OS and DFS in PDAC cohort from GEPIA database. OASL, oligoadenylate synthetases-like; TPM, transcripts per million; OS, overall survival; DSS, disease-specific survival; PDAC, pancreatic ductal adenocarcinoma; DFS, disease-free survival; TCGA, The Cancer Genome Atlas; GEPIA, Gene Expression Profile Interactive Analysis.

Table 4 The results of univariate and multivariable cox regression analyses

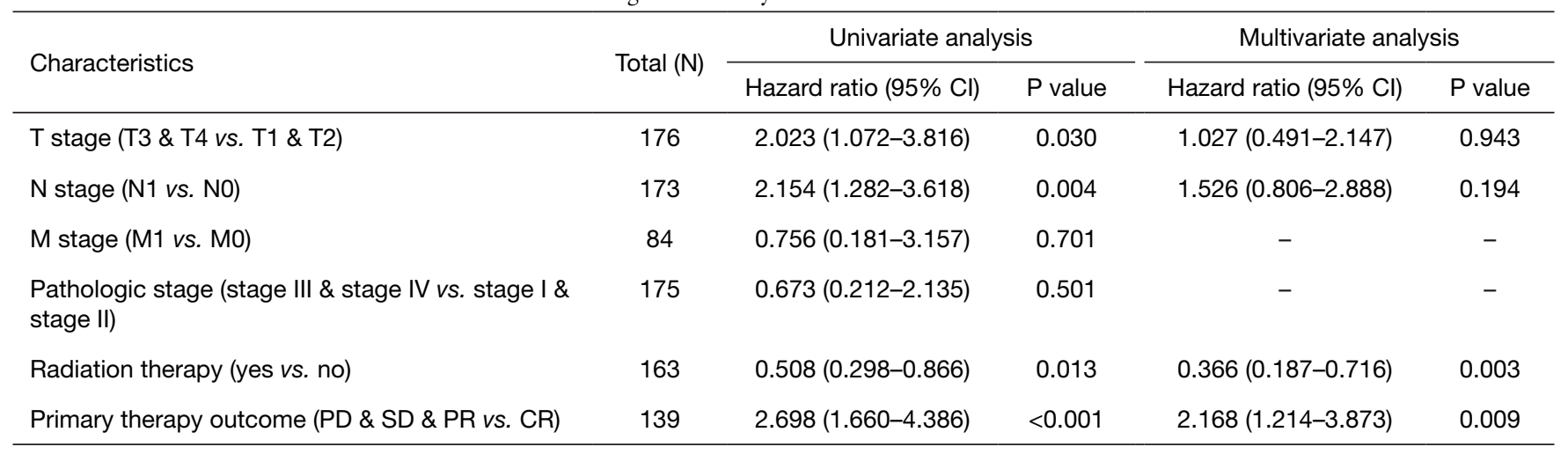

Table 4 (continued) 
Table 4 (continued)

\begin{tabular}{|c|c|c|c|c|c|}
\hline Characteristics & Total $(\mathrm{N})$ & \multicolumn{2}{|c|}{ Univariate analysis } & \multicolumn{2}{|c|}{ Multivariate analysis } \\
\hline Gender (male vs. female) & 178 & $0.809(0.537-1.219)$ & 0.311 & - & - \\
\hline Race (Black or African American \& White vs. Asian) & 174 & $1.254(0.507-3.099)$ & 0.624 & - & - \\
\hline Age (>65 vs. $\leq 65)$ & 178 & $1.290(0.854-1.948)$ & 0.227 & - & - \\
\hline Histologic grade (G3 \& G4 vs. G1 \& G2) & 176 & $1.538(0.996-2.376)$ & 0.052 & $1.993(1.149-3.456)$ & 0.014 \\
\hline $\begin{array}{l}\text { Anatomic neoplasm subdivision (other vs. head of } \\
\text { pancreas) }\end{array}$ & 178 & $0.417(0.231-0.754)$ & 0.004 & $0.473(0.222-1.008)$ & 0.053 \\
\hline Smoker (yes vs. no) & 144 & $1.086(0.687-1.719)$ & 0.724 & - & - \\
\hline OASL (high vs. low) & 178 & $1.810(1.189-2.754)$ & 0.006 & $1.750(1.056-2.900)$ & 0.030 \\
\hline
\end{tabular}

$\mathrm{Cl}$, confidence interval; PD, progressive disease; SD, stable disease; PR, partial remission; CR, complete remission; OASL, oligoadenylate synthetases-like.

Points
Radiation therapy
Primary therapy outcome
Histologic grade
OASL
Total points
Linear predictor
1-year survival probability
3-year survival probability
5-year survival probability

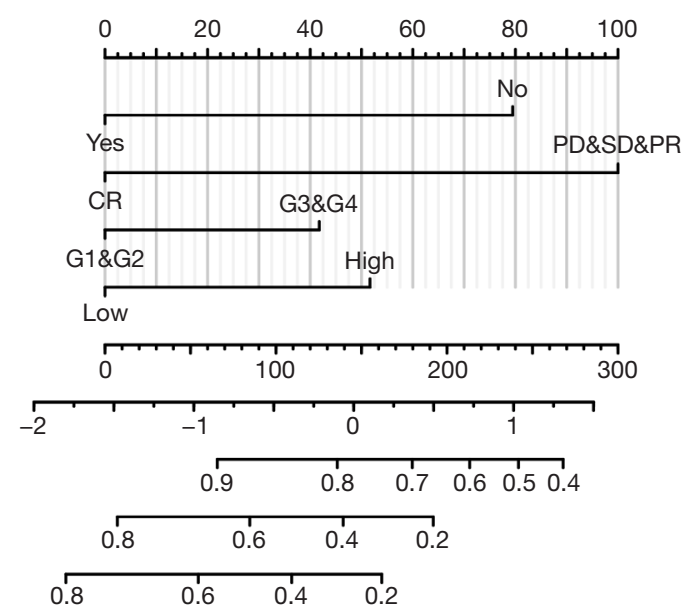

Figure 4 Nomogram for predicting the survival probability. OASL, oligoadenylate synthetases-like; CR, complete remission; PD, progressive disease; SD, stable disease; PR, partial remission.

and metastasis. After the knockdown of OASL, we found that the proliferation and invasion of cells were inhibited, and the apoptosis rate increased. We speculate that the high expression of OASL may promote the malignant biological behavior of PDAC cells, resulting in the poor prognosis of patients. Nevertheless, further research is needed on which pathways OASL affects the phenotype of cells and whether it can be used as a target of chemoresistance.
In conclusion, our study shows that OASL can be used as a prognostic biomarker and a potential therapeutic target in PDAC.

\section{Conclusions}

The expression of OASL has been associated with immune infiltrating cells. Our study investigated the role of OASL 


\begin{tabular}{ccccc}
\hline Characteristics & \multicolumn{2}{c}{ Total( $\mathrm{N}) \mathrm{HR}(95 \% \mathrm{CI})$ Univariate analysis } & P value Univariate analysis \\
\hline T stage & 176 & $2.023(1.072-3.816)$ & 0.03 \\
N stage & 173 & $2.154(1.282-3.618)$ & 0.004 \\
Radiation therapy & 163 & $0.508(0.298-0.866)$ & $<0.001$ \\
Primaty therapy outcome & 139 & $2.698(1.660-4.386)$ & 0.028 \\
Residual tumor & 164 & $1.645(1.056-2.561)$ & 0.004 \\
Anatomicneoplasm subdivision & 178 & $0.417(0.231-0.754)$ & $1.810(1.189-2.754)$ & 0.006 \\
OASL & 178 & & 1 & \\
\hline
\end{tabular}

Figure 5 Multivariate Cox analysis of OASL expression and other clinicopathological factors. T stage, $\mathrm{N}$ stage, radiation therapy, primary therapy outcome, residual tumor, and OASL are independent prognostic factors. OASL, oligoadenylate synthetases-like; CI, confidence interval; HR, hazard ratio.
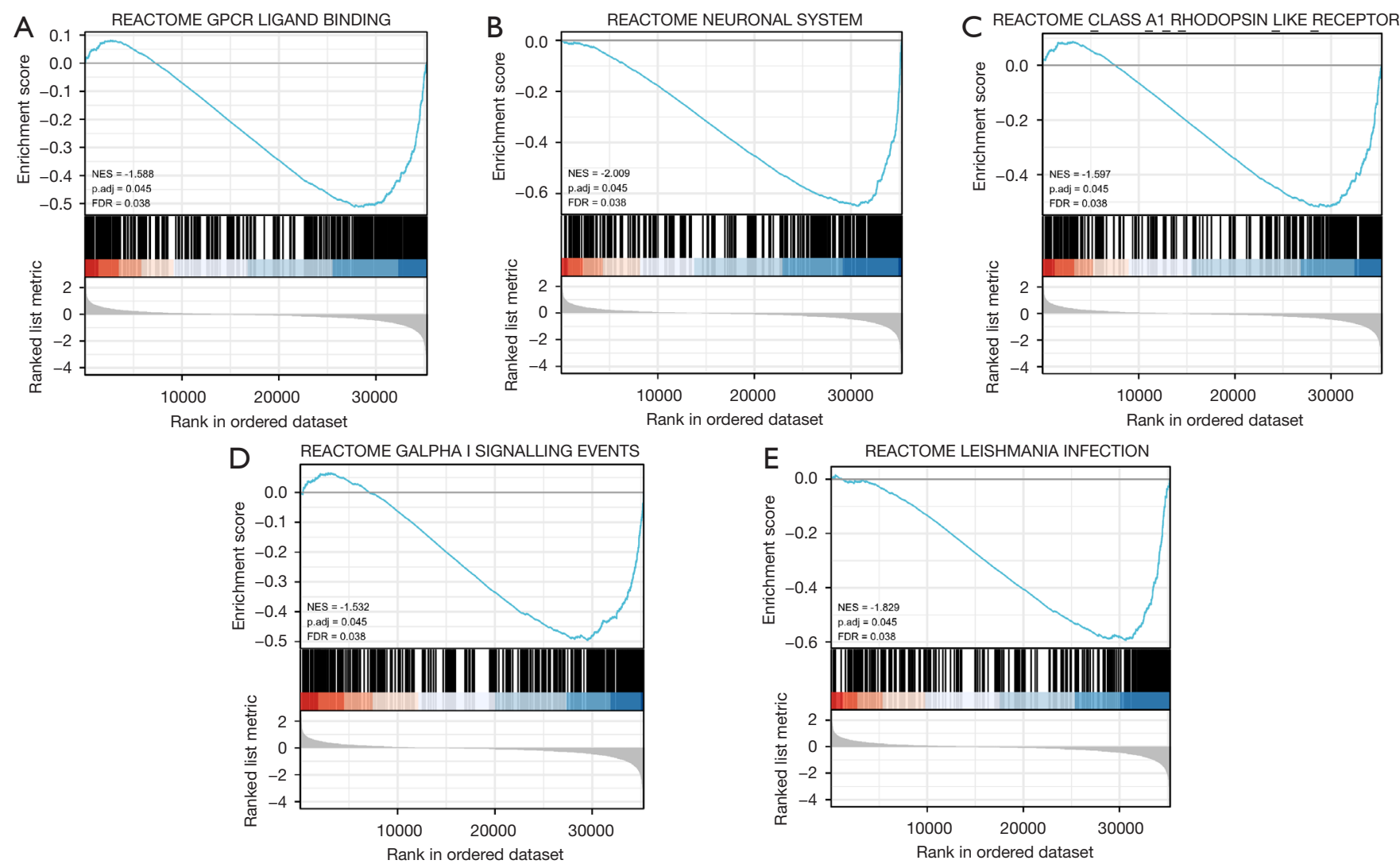

Figure 6 GSEA of OASL in PDAC. (A) GPCR-ligand binding. (B) Neuronal system. (C) Class A/1 (rhodopsin-like receptors). (D) G-Alpha/1 signaling events. (E) Leishmania infection were enriched in OASL-related PDAC. GSEA, Gene Set Enrichment Analysis; OASL, oligoadenylate synthetases-like; PDAC, pancreatic ductal adenocarcinoma; NES, normalized ES; FDR, false discovery rate. 

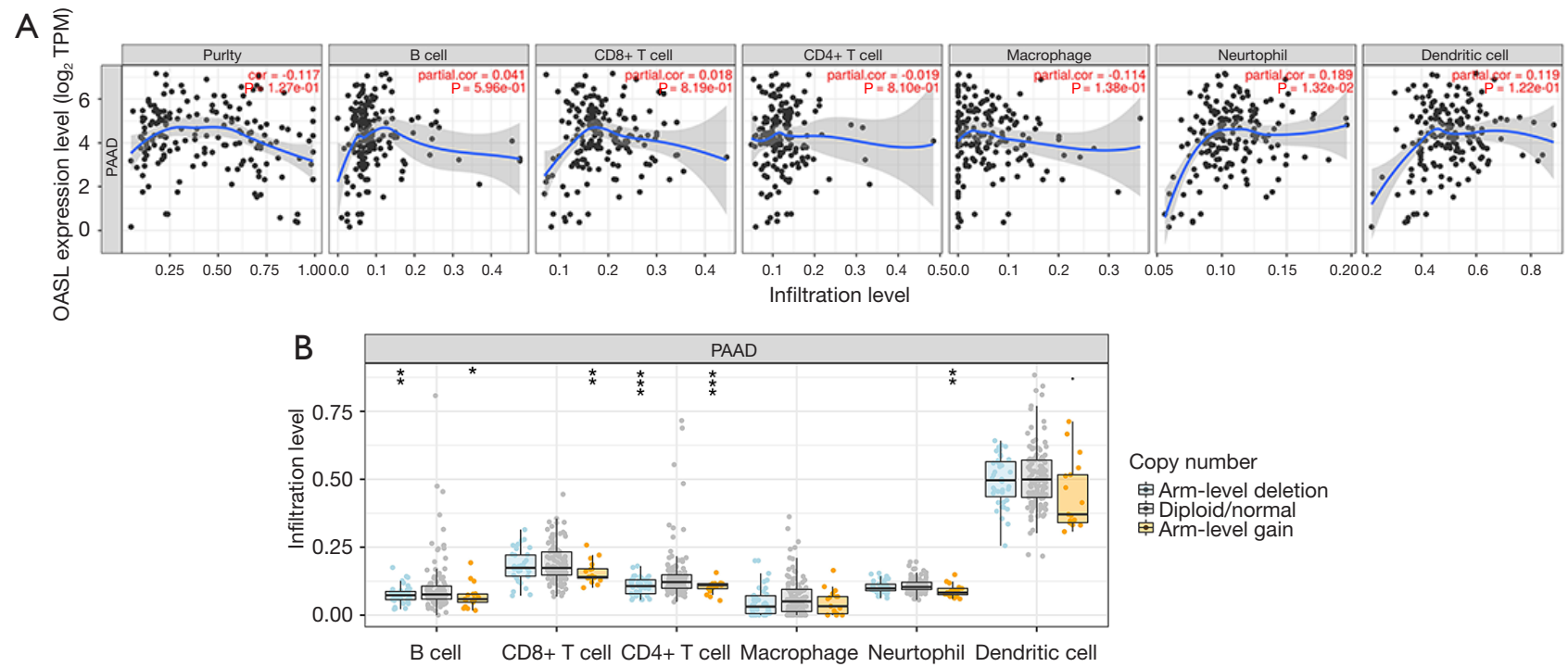

Copy number

由 Arm-level deletion ( Diploid/normal 由 Arm-level gain

Figure 7 Correlation analysis of OASL expression and infiltration levels of immune cells in PDAC tissues using the TIMER. (A) OASL expression level has significant positive correlations with infiltrating levels of Neutrophils ( $r=0.189, \mathrm{P}=1.32 \mathrm{e}-02)$. (B) The infiltration level of various immune cells under different copy numbers of OASL in PDAC. * $\mathrm{P}<0.05$. **, $\mathrm{P}<0.01$. ***, $\mathrm{P}<0.001$. OASL, oligoadenylate synthetases-like; TPM, transcripts per million; PAAD, pancreatic adenocarcinoma; PDAC, pancreatic ductal adenocarcinoma; TIMER, Tumor Immune Estimation Resource.
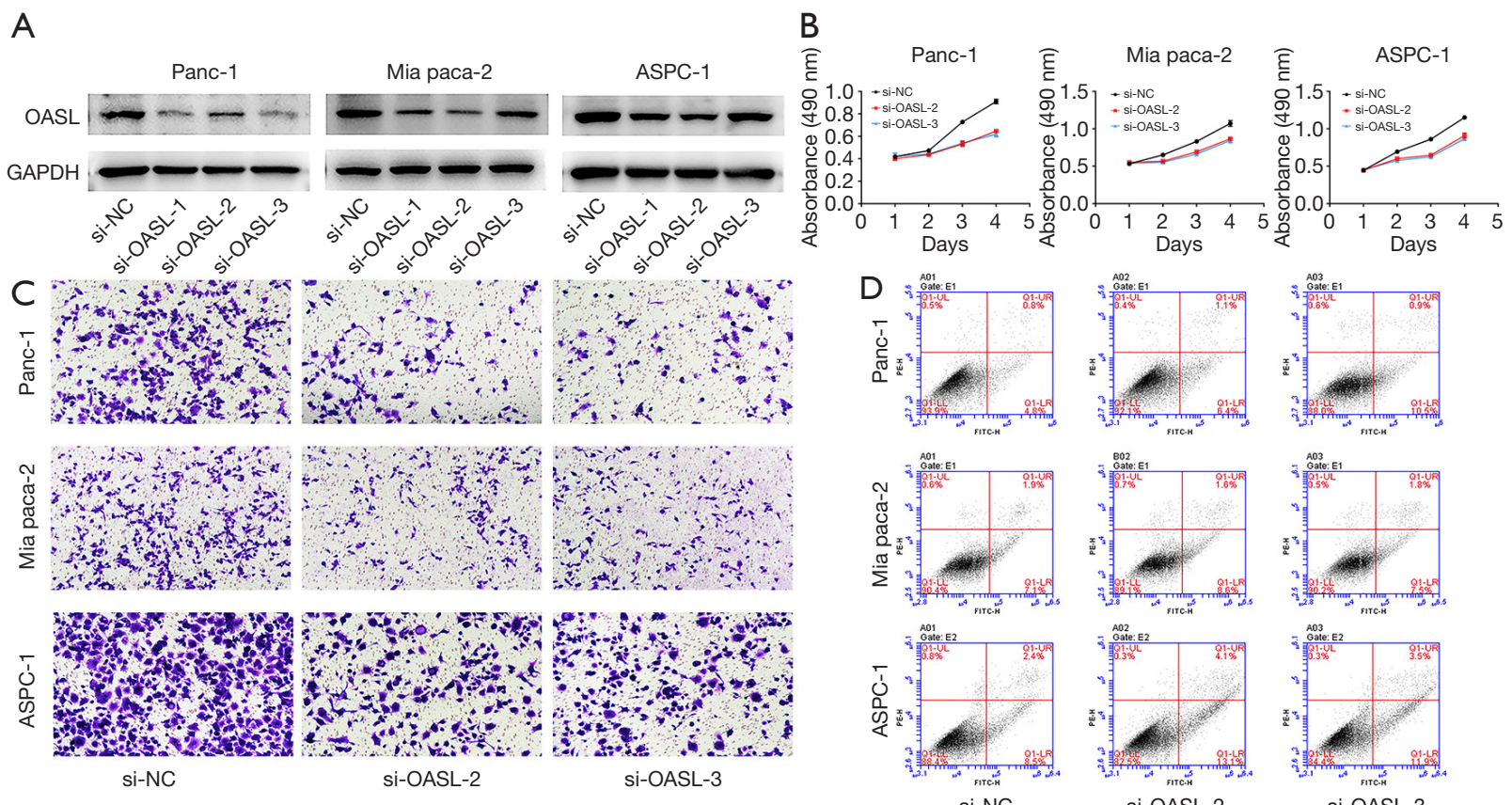

Figure 8 Knock-down of OASL inhibits cells proliferation, invasion and promotes apoptosis of PDAC cells. (A) The OASL protein expression was significantly decreased upon si-OASL transfection. (B) Cell viability was measured by MTT assay (Panc-1, si-NC vs. siOASL-1. $\mathrm{P}<0.05$. si-NC vs. si-OASL-2. $\mathrm{P}<0.05$. Mia paca-2, si-NC vs. si-OASL-1. $\mathrm{P}<0.05$. si-NC vs. si-OASL-2. $\mathrm{P}<0.05$. Aspc-1, si-NC vs. si-OASL-1. $\mathrm{P}<0.05$. si-NC vs. si-OASL-2. $\mathrm{P}<0.05$ ). (C) The invasive ability of si-OASL groups appeared sharply reduced in the Transwell assay. Crystal violet-stained invasive cells were captured using an inverted microscope at $\times 100$ magnification. (D) Obviously more apoptosis was observed in si-OASL groups. Experiments were repeated a minimum of 3 times. OASL, oligoadenylate synthetases-like; PDAC, pancreatic ductal adenocarcinoma; MTT, 3-(4,5-dimethylthiazol-2-yl)-2,5-diphenyltetrazolium bromide. 
in PDAC and provided novel prognostic biomarkers and therapeutic targets for PDAC.

\section{Acknowledgments}

Funding: This work was supported by Natural Science Foundation of Shandong Province, China (ZR2017MH032); Key Technology Research and Development Program of Shandong (2019GSF108065); Natural Science Foundation of Shandong Province, China (ZR2020MH256); Key Technology Research and Development Program of Shandong (2019GSF108254); the Medical Health Science and Technology Project of Shandong Provincial Health Commission (2019WS386); The National Natural Science Foundation of China (Grant No. 81900731) and ECCM Program of Clinical Research Center of Shandong University (No. 2021SDUCRCB010).

\section{Footnote}

Reporting Checklist: The authors have completed the REMARK reporting checklist. Available at https://atm. amegroups.com/article/view/10.21037/atm-21-6618/rc

Data Sharing Statement: Available at https://atm.amegroups. com/article/view/10.21037/atm-21-6618/dss

Conflicts of Interest: All authors have completed the ICMJE uniform disclosure form (available at https://atm. amegroups.com/article/view/10.21037/atm-21-6618/coif). The authors have no conflicts of interest to declare.

Ethical Statement: The authors are accountable for all aspects of the work in ensuring that questions related to the accuracy or integrity of any part of the work are appropriately investigated and resolved. The study was conducted in accordance with the Declaration of Helsinki (as revised in 2013).

Open Access Statement: This is an Open Access article distributed in accordance with the Creative Commons Attribution-NonCommercial-NoDerivs 4.0 International License (CC BY-NC-ND 4.0), which permits the noncommercial replication and distribution of the article with the strict proviso that no changes or edits are made and the original work is properly cited (including links to both the formal publication through the relevant DOI and the license).
See: https://creativecommons.org/licenses/by-nc-nd/4.0/.

\section{References}

1. Hammel P, Kindler HL, Reni M, et al. Health-related quality of life in patients with a germline BRCA mutation and metastatic pancreatic cancer receiving maintenance olaparib. Ann Oncol 2019;30:1959-68.

2. Shu X, Zheng W, Yu D, et al. Prospective metabolomics study identifies potential novel blood metabolites associated with pancreatic cancer risk. Int J Cancer 2018;143:2161-7.

3. Kamisawa T, Wood LD, Itoi T, et al. Pancreatic cancer. Lancet 2016;388:73-85.

4. Jiang H, Liu X, Knolhoff BL, et al. Development of resistance to FAK inhibition in pancreatic cancer is linked to stromal depletion. Gut 2020;69:122-32.

5. Ilic M, Ilic I. Epidemiology of pancreatic cancer. World J Gastroenterol 2016;22:9694-705.

6. Qian Y, Gong Y, Fan Z, et al. Molecular alterations and targeted therapy in pancreatic ductal adenocarcinoma. J Hematol Oncol 2020;13:130.

7. Perkhofer L, Gout J, Roger E, et al. DNA damage repair as a target in pancreatic cancer: state-of-the-art and future perspectives. Gut 2021;70:606-17.

8. Lee MS, Kim B, Oh GT, et al. OASL1 inhibits translation of the type I interferon-regulating transcription factor IRF7. Nat Immunol 2013;14:346-55.

9. Sim CK, Cho YS, Kim BS, et al. 2'-5' Oligoadenylate synthetase-like 1 (OASL1) deficiency in mice promotes an effective anti-tumor immune response by enhancing the production of type I interferons. Cancer Immunol Immunother 2016;65:663-75.

10. Muhammad SA, Guo J, Nguyen TM, et al. Simulation Study of cDNA Dataset to Investigate Possible Association of Differentially Expressed Genes of Human THP1Monocytic Cells in Cancer Progression Affected by Bacterial Shiga Toxins. Front Microbiol 2018;9:380.

11. Yu C, Xue P, Zhang L, et al. Prediction of key genes and pathways involved in trastuzumab-resistant gastric cancer. World J Surg Oncol 2018;16:174.

12. Zhang Y, Yu C. Prognostic characterization of OAS1/ OAS2/OAS3/OASL in breast cancer. BMC Cancer 2020;20:575.

13. Lv J, Wang L, Shen H, et al. Regulatory roles of OASL in lung cancer cell sensitivity to Actinidia chinensis Planch root extract (acRoots). Cell Biol Toxicol 2018;34:207-18.

14. Rhodes DR, Kalyana-Sundaram S, Mahavisno V, et 
al. Oncomine 3.0: genes, pathways, and networks in a collection of 18,000 cancer gene expression profiles. Neoplasia 2007;9:166-80.

15. Tang Z, Li C, Kang B, et al. GEPIA: a web server for cancer and normal gene expression profiling and interactive analyses. Nucleic Acids Res 2017;45:W98-W102.

16. Vivian J, Rao AA, Nothaft FA, et al. Toil enables reproducible, open source, big biomedical data analyses. Nat Biotechnol 2017;35:314-6.

17. Li T, Fan J, Wang B, et al. TIMER: A Web Server for Comprehensive Analysis of Tumor-Infiltrating Immune Cells. Cancer Res 2017;77:e108-10.

18. Seshacharyulu P, Baine MJ, Souchek JJ, et al. Biological determinants of radioresistance and their remediation in pancreatic cancer. Biochim Biophys Acta Rev Cancer 2017;1868:69-92.

19. Singhi AD, Koay EJ, Chari ST, et al. Early Detection of Pancreatic Cancer: Opportunities and Challenges. Gastroenterology 2019;156:2024-40.

20. Yin F, Hu K, Chen Y, et al. SiRNA Delivery with PEGylated Graphene Oxide Nanosheets for Combined Photothermal and Genetherapy for Pancreatic Cancer. Theranostics 2017;7:1133-48.

21. Chang JC, Kundranda M. Novel Diagnostic and Predictive Biomarkers in Pancreatic Adenocarcinoma. Int J Mol Sci 2017;18:667.

22. Fagone P, Nunnari G, Lazzara F, et al. Induction of OAS gene family in HIV monocyte infected patients with high and low viral load. Antiviral Res 2016;131:66-73.

23. Zhang W, Cao X, Cao G, et al. OASs in Defense of Mycobacterial Infection: Angels or Demons? Curr Issues Mol Biol 2021;40:221-30.

Cite this article as: Chen S, Sun Z, Zhao W, Meng M, Guo W, Wu D, Shu Q, Chai J, Wang L. Oligoadenylate synthetases-like is a prognostic biomarker and therapeutic target in pancreatic ductal adenocarcinoma. Ann Transl Med 2022;10(3):138. doi: 10.21037/atm-21-6618
24. Feng X, Huang J, Liu Y, et al. Identification of interferoninducible genes as diagnostic biomarker for systemic lupus erythematosus. Clin Rheumatol 2015;34:71-9.

25. Xiang Y, Zhou S, Hao J, et al. Development and validation of a prognostic model for kidney renal clear cell carcinoma based on RNA binding protein expression. Aging (Albany NY) 2020;12:25356-72.

26. Zhang L, Jiang Y, Lu X, et al. Genomic characterization of cervical cancer based on human papillomavirus status. Gynecol Oncol 2019;152:629-37.

27. Stopa KB, Kusiak AA, Szopa MD, et al. Pancreatic Cancer and Its Microenvironment-Recent Advances and Current Controversies. Int J Mol Sci 2020;21:3218.

28. Ho WJ, Jaffee EM, Zheng L. The tumour microenvironment in pancreatic cancer - clinical challenges and opportunities. Nat Rev Clin Oncol 2020;17:527-40.

29. Zhang C, Zou Y, Zhu Y, et al. Three Immune-Related Prognostic mRNAs as Therapeutic Targets for Pancreatic Cancer. Front Med (Lausanne) 2021;8:649326.

30. Brandau S, Dumitru CA, Lang S. Protumor and antitumor functions of neutrophil granulocytes. Semin Immunopathol 2013;35:163-76.

31. Xiang ZJ, Hu T, Wang Y, et al. Neutrophillymphocyte ratio (NLR) was associated with prognosis and immunomodulatory in patients with pancreatic ductal adenocarcinoma (PDAC). Biosci Rep 2020;40:BSR20201190.

32. Huber M, Brehm CU, Gress TM, et al. The Immune Microenvironment in Pancreatic Cancer. Int J Mol Sci 2020;21:7307.

(English Language Editor: J. Jones) 